\title{
Accurate Schemes for Advection and Aeroacoustics
}

\author{
Cheolwan Kim* and Philip L. Roe ${ }^{\dagger}$ \\ W. M. Keck Foundation Lab for Computational Fluid Dynamics \\ Department of Aerospace Engineering, \\ The University of Michigan, Ann Arbor, MI 48109-2140 \\ Jeffrey P. Thomas ${ }^{\ddagger}$ \\ Department of Mechanical Engineering and Materials Science \\ Duke University, 90300 Durham, NC 27708-0300
}

\begin{abstract}
Higher order upwind leapfrog schemes for two dimensional advection, acoustics and aeroacoustics are developed and applied to several examples. Advection schemes are applied to scalar advection with uniform and nonuniform creeping flow speed. Acoustics schemes are implemented successfully in polar coordinates with new damping technique. Increasing the order of accuracy for Aeroacoustics schemes was achieved and implemented to the reflection of acoustic pulse problem.
\end{abstract}

\section{Introduction}

In this paper we continue a sequence of developments $[1,2,3]$ of Iserles' 'generalised' or 'upwind' leapfrog schemes [4]. The philosophy on which these schemes are based is to avoid numerical dissipation by constructing them to be time-reversible, but the symmetry that ensures this is a skew symmetry with regard to the centroid of the stencil, rather than (as with conventional leapfrog schemes) a strict symmetry in both space and time.

Implementing this strategy calls for different special measures in the cases of advection and wave motion. For advection the coefficients of the scheme change discontinuously as the advection speed switches from one quadrant to another unless the schemes appropriate to each quadrant are suitably blended. Section 2 describes schemes of both second- and fourth-order accuracy, and shows that they can indeed be blended successfully. For wave propagation problems, it is possible to retain reversibility, but only by adopting a strategy of staggered storage that involves edge-based variables. For this reason, edge-based storage is used in the advection schemes

\footnotetext{
*Doctoral Candidate,

†Professor, AIAA Fellow

'Research Assistant Professor, AIAA member
}

Copyright (C) 1997 by the American Institute of Aeronautics and Astronautics, Inc. All rights reserved. also. Section 3 discusses schemes for pure wave problems, and Section 4 combines wave propogation with advection (the aeroacoustic problem).

\section{Two Dimensional Advection Equation}

For two dimensional advection equation,

$$
u_{t}+a u_{x}+b u_{y}=0
$$

two of the simplest choices of stencil that follow the above principles are shown in Fig1. The first one is the three level compact scheme with staggered grid(storage at cell edges). It is a time reversible second-order one and the most compact scheme we can imagine.

$$
u_{p}=u_{d}+(1-2 \nu)\left(u_{1}-u_{2}\right)-2 \mu\left(u_{3}-u_{4}\right)
$$

where $\nu=a \Delta t / \Delta x$ and $\mu=b \Delta t / \Delta y$. The other one, shown in Fig1.b is a three level time reversible scheme with node-based storage. It attains also second order accuracy but it is extended into $y$-direction.

$$
u_{p}=u_{d}+(1-2 \nu)\left(u_{2}-u_{5}\right)-\frac{\mu}{2}\left(u_{1}-u_{3}+u_{4}-u_{6}\right)
$$

\subsection{Fourth-order advection schemes}

Although the second order schemes are compact and very easy to implement, their accuracy is not sufficient to simulate long-range advection problem and they require very fine grids to obtain reasonable accuracy. Therefore it is indispensible to increase the accuracy to fourth or sixth order. With higher order scheme, if grid size is reduced by a factor $k$ in each direction, the storage for a $n$-dimensional problem will be reduced by a factor $k^{n}$ and cpu by $k^{n+1}$. To raise the order of ac- 


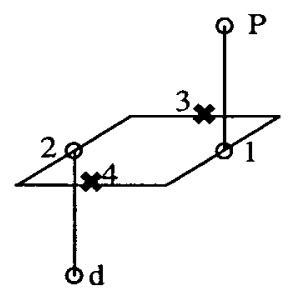

(a)

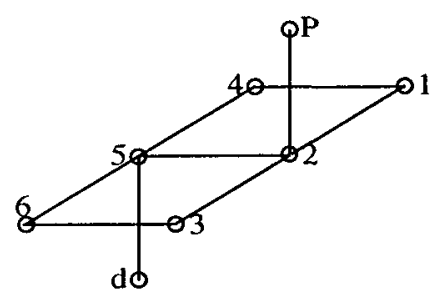

(b)

Figure 1: Three time level stencils for two dimensional advection equation. (a) Staggered grid (b) Uniform Grid
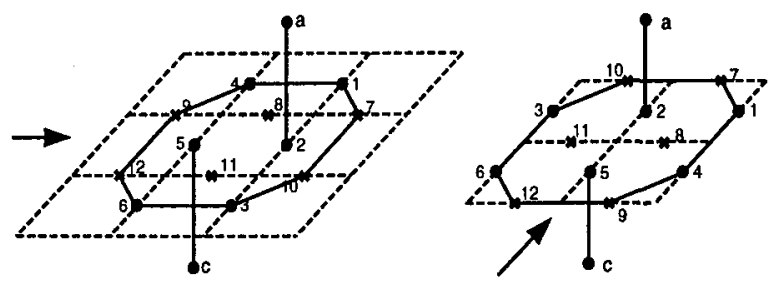

Figure 2: Three time level fourth-order stencils for two dimensional advection equation

curacy to four or six, the stencil could be extended in either the space or time domain. Figure 2 shows fourth-order time reversible stencils for advection equations, just extended in space. Scheme(a) is appropriate for a propagation direction aligned with $+x$, say $-\pi / 4<\alpha<\pi / 4, \alpha=\tan ^{-1}(b / a)$. Scheme(b) is with $+\mathrm{y}$, say $\pi / 4<\alpha<3 / 4 \pi$. They are quite compact, but require special treatment at boundaries.

Scheme (a)

$$
\begin{aligned}
u_{a}= & u_{c}+(1-2 \nu)\left(u_{2}-u_{5}\right)-2 \mu\left(u_{8}-u_{1} 1\right) \\
& -C_{1}\left[2\left(u_{7}+u_{10}-u_{9}-u_{12}\right)-\left(u_{1}+6 u_{2}+u_{3}\right)\right. \\
& \left.+\left(u_{4}+6 u_{5}+u_{6}\right)\right]-C_{2}\left[2\left(u_{1}+u_{4}-u_{3}-u_{6}\right)\right. \\
& \left.-\left(u_{7}+6 u_{8}+u_{9}\right)+\left(u_{10}+6 u_{11}+u_{12}\right)\right] \\
& -C_{3}\left[\left(u_{7}-2 u_{8}+u_{9}\right)-\left(u_{10}-2 u_{11}+u_{12}\right)\right] \\
& -C_{4}\left[\left(u_{1}-2 u_{2}+u_{3}\right)-\left(u_{4}-2 u_{5}+u_{6}\right)\right]
\end{aligned}
$$

where

$$
\begin{array}{ll}
C_{1}=\frac{1}{6} \nu(\nu-1)(2 \nu-1), & C_{2}=\frac{1}{12} \mu(2 \nu-1)(2 \nu+1) \\
C_{3}=\frac{1}{4} \mu(2 \nu-1)^{2}, & C_{4}=\frac{1}{2} \mu^{2}(2 \nu-1)
\end{array}
$$

Scheme (b)

$$
\begin{aligned}
u_{a}= & u_{c}+(1-2 \mu)\left(u_{2}-u_{5}\right)-2 \nu\left(u_{8}-u_{1} 1\right) \\
& -C_{1}\left[2\left(u_{1}+u_{4}-u_{3}-u_{6}\right)-\left(u_{7}+6 u_{8}+u_{9}\right)\right. \\
& \left.+\left(u_{10}+6 u_{11}+u_{12}\right)\right]-C_{2}\left[2\left(u_{7}+u_{10}-u_{9}-u_{12}\right)\right. \\
& \left.-\left(u_{1}+6 u_{2}+u_{3}\right)+\left(u_{4}+6 u_{5}+u_{6}\right)\right]
\end{aligned}
$$
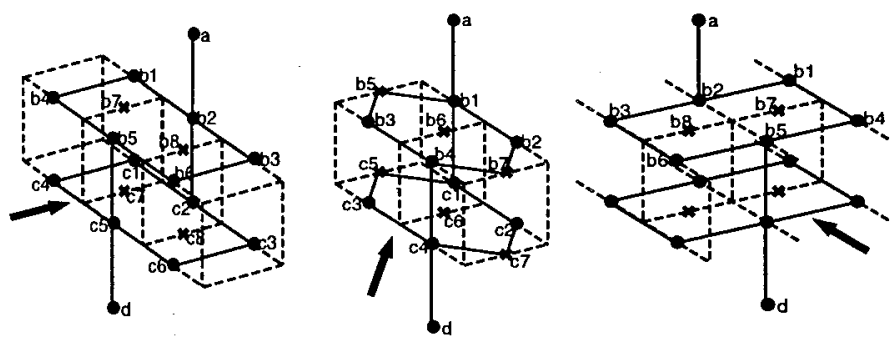

Figure 3: Four time level fourth-order stencils for two dimensional advection equation

$$
\begin{aligned}
& -C_{3}\left[\left(u_{1}-2 u_{2}+u_{3}\right)-\left(u_{4}-2 u_{5}+u_{6}\right)\right] \\
& -C_{4}\left[\left(u_{7}-2 u_{8}+u_{9}\right)-\left(u_{10}-2 u_{11}+u_{12}\right)\right]
\end{aligned}
$$

where

$$
\begin{array}{ll}
C_{1}=\frac{1}{12} \nu(2 \nu-1)(2 \nu+1), & C_{2}=\frac{1}{6} \mu(\mu-1)(2 \mu-1) \\
C_{3}=\frac{1}{2} \nu^{2}(2 \mu-1), & C_{4}=\frac{1}{4} \nu(2 \mu-1)^{2}
\end{array}
$$

Schemes(a), (b) must be blended if $\alpha$ is close to $\pm \pi / 4$ (see section 2.2). Figure 3 shows another set of fourth-order time reversible stencil. They are four level and extended in space slightly. Scheme(a), (b) and (c) each are adequate for $\mathrm{x}$-direction, oblique and $\mathrm{y}$-direction advection. Again a blending function is used for nonconstant advection directions.

Scheme (a)

$$
\begin{aligned}
& u_{a}=u_{b 2}-\left(u_{c 5}-u_{d}\right) \\
& -C_{1}\left(u_{b 2}-u_{c 2}+u_{b 5}-u_{c 5}\right)-C_{2}\left(u_{b 2}-u_{b 5}+u_{c 2}-u_{c 5}\right) \\
& -C_{3}\left(u_{b 1}-u_{b 4}+u_{c 3}-u_{c 4}\right)-C_{4}\left(u_{b 3}-u_{b 6}+u_{c 1}-u_{c 4}\right) \\
& -C_{5}\left(u_{b 7}-u_{b 8}+u_{c 7}-u_{c 8}\right)-C_{6}\left(u_{b 1}-u_{b 3}+u_{c 4}-u_{c 6}\right)
\end{aligned}
$$

where

$$
\begin{aligned}
& C_{1}=(5 \nu-1) /(\nu+1) \\
& C_{2}=\left[\nu\left(6 \nu^{2}+\mu^{2}\right)-\mu^{2}\left(\nu^{2}+\mu^{2}\right)\right] / \nu(\nu+1) \\
& C_{3}=\mu^{2}(\nu-\mu)(\nu-\mu+1) / 2 \nu(\nu+1) \\
& C_{4}=\mu^{2}(\nu+\mu)(\nu+\mu-1) / 2 \nu(\nu+1) \\
& C_{5}=4 \mu\left(2 \nu-\mu^{2}\right) /(\nu+1) \\
& C_{6}=\mu\left(2 \mu^{2}-\nu\right) /(\nu+1)
\end{aligned}
$$

Scheme (b)

$$
\begin{aligned}
& u_{a}=u_{b 1}-\left(u_{c 4}-u_{d}\right)-C_{1}\left(u_{b 1}-u_{c 1}+u_{b 4}-u_{c 4}\right) \\
& \quad-C_{2}\left(u_{b 1}-u_{b 3}+u_{c 2}-u_{c 4}\right)-C_{3}\left(u_{b 2}-u_{b 4}+u_{c 1}-u_{c 3}\right) \\
& -C_{4}\left(u_{b 5}-u_{b 6}+u_{c 6}-u_{c 7}\right)-C_{5}\left(u_{b 6}-u_{b 7}+u_{c 5}-u_{c 6}\right) \\
& -C_{6}\left(u_{b 1}-u_{b 2}+u_{b 3}-u_{b 4}+u_{c 1}-u_{c 2}+u_{c 3}-u_{c 4}\right)
\end{aligned}
$$

where

$$
C_{1}=(5 \nu-1) /(\nu+1)
$$




$$
\begin{aligned}
& C_{2}=[(\nu-1)(4 \nu-\mu)+2 \nu] /(\nu+1) \\
& C_{3}=[2 \nu(\nu+1)+\mu(\nu-1)] /(\nu+1) \\
& C_{4}=\mu(3 \nu-\mu+1) /(\nu+1) \\
& C_{5}=\mu(\nu+\mu-1) /(\nu+1) \\
& C_{6}=\nu \mu /(\nu+1)
\end{aligned}
$$

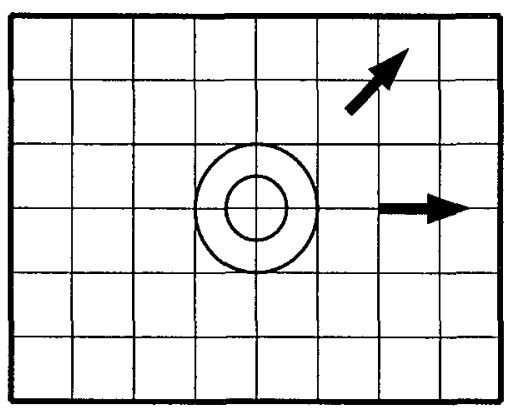

scheme (c)

$$
\begin{aligned}
& u_{a}=u_{b 2}-\left(u_{c 5}-u_{d}\right) \\
& \quad-C_{1}\left(u_{b 2}-u_{c 2}+u_{b 5}-u_{c 5}\right)-C_{2}\left(u_{b 7}-u_{b 8}+u_{c 7}-u_{c 8}\right) \\
& \quad-C_{3}\left(u_{b 1}-u_{b 3}+u_{c 4}-u_{c 6}\right)-C_{4}\left(u_{b 2}-u_{b 5}+u_{c 2}-u_{c 5}\right) \text { Figure 4: Illustration of the test case for accuracy com- }
\end{aligned}
$$$$
-C_{5}\left(u_{b 1}-u_{b 4}+u_{c 3}-u_{c 4}\right)-C_{6}\left(u_{b 3}-u_{b 6}+u_{c 1}-u_{c 4}\right) \text { parison of fourth-order advection schemes }
$$

where

$$
\begin{aligned}
& C_{1}=(5 \mu-1) /(\mu+1) \\
& C_{2}=4 \nu\left(2 \mu-\nu^{2}\right) /(\mu+1) \\
& C_{3}=\nu\left(2 \nu^{2}-\mu\right) /(\mu+1) \\
& C_{4}=\left[\mu\left(6 \mu^{2}+\nu^{2}\right)-\nu^{2}\left(\nu^{2}+\mu^{2}\right)\right] / \mu(\mu+1) \\
& C_{5}=\nu^{2}(\mu-\nu)(\mu-\nu-1) / 2 \mu(\mu+1) \\
& C_{6}=\nu^{2}(\mu+\nu)(\mu+\nu-1) / 2 \mu(\mu+1)
\end{aligned}
$$

\subsection{Blending Function}

Choosing schemes between (a) and (b) in Figure 2 depends on the advection direction and either one or some combination of these schemes are chosen. For $\mathrm{x}$-direction advection, scheme(a) is used and scheme(b) is for $y$ direction. If the advection direction is oblique, a combination of scheme(a) and (b) is used to transit from one to another continuously. To blend two schemes will introduce slight dissipation and the dissipation rate is proportional to $d \phi^{2}$, where $d \phi$ is the phase difference of two updated scalars, i.e. it is eighth-order for the fourthorder schemes. If $\alpha$ is less than $40^{\circ}$ scheme(a) is used, and scheme(b) for $\alpha>50^{\circ}$. If $\alpha$ is between $40^{\circ}$ and $50^{\circ}$, it is necessary to blend scheme(a) and scheme(b). The blending function is selected as

$$
\begin{aligned}
u_{n e w} & =\frac{1}{2}\left[\left(u_{a}^{n e w}+u_{b}^{n e w}\right)-r(\alpha)\left(u_{a}^{n e w}-u_{b}^{n e w}\right)\right] \\
r(\alpha) & =\sin \left(0.1 \pi\left(\alpha-45^{\circ}\right)\right)
\end{aligned}
$$

Both schemes update the variable very accurately in the transition area, $d \phi$ is very small and dissipation, proportional to $d \phi^{2}$, is much smaller. For four level schemes, Figure 3 , blending function is needed at the transition area. When $\alpha$ is between $30^{\circ}$ and $35^{\circ}$ scheme(a) and (b) are blended and scheme(b) and (c) blended for $60^{\circ}<\alpha<65^{\circ}$.

\subsection{Numerical Experiment}

First of all, to compare the accuracy of the above, a simple test problem, shown in Figure 4 is chosen. A Gaussian distribution, $u(x, y)=\exp \left(-\ln 2 / 0.2^{2}\left(x^{2}+y^{2}\right)\right)$ on

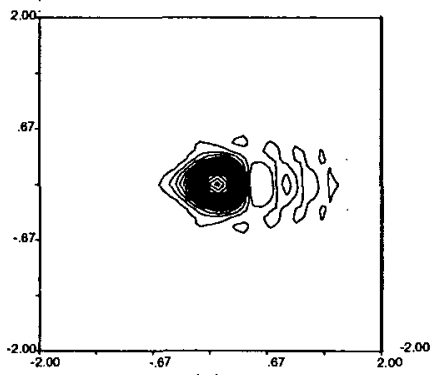

(a)

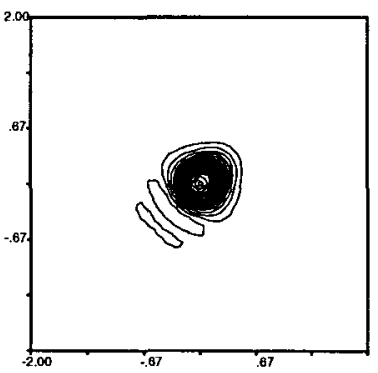

(b)

Figure 5: Three level scheme accuracy test result (a)advection along $\mathrm{x}$-direction (b)advection oblique to $\mathrm{x}$-direction, $\alpha=45.0^{\circ}$

coarse grid is advected parallel and oblique to the grid line. The grid size is $\Delta x=\Delta y=0.1$ and time step is $\Delta t=0.04$. Numerical results after 5,000 iteration are shown in Figure 5 and Figure 6. The distribution revolved through computational domain 50 times. Figure 5 shows the results updated by three level scheme. The first result, Figure 5.a shows leading errors and peak value is decreased to 82.7 percent of initial profile. Figure 5 .b shows lagging errors and its peak value is 82 percent of the exact one. The extra dissipation due to blending the two schemes is neglible. Figure 6 is the results of the four level schemes. Figure 6.(a) is preserving the initial profile extraordinarily well and Figure 6.(b) shows slight lagging errors in the $y$-direction; its peak value is 82 percent of the exact one. It is updated with two adjacent schemes and blended slightly. Both schemes used for this computation have very accurate phase property in $x$-direction and show lagging error in $y$-direction. To demonstrate the method on a polar grid and with non-uniform velocities, we considered a scalar distribution advected with the speed of creeping flow around a circular cylinder. That is, we solved.

$$
u_{t}+a_{r} \cdot u_{r}+a_{\theta} \frac{1}{r} u_{\theta}=0
$$




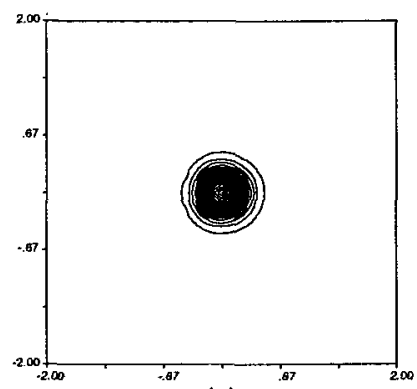

(a)

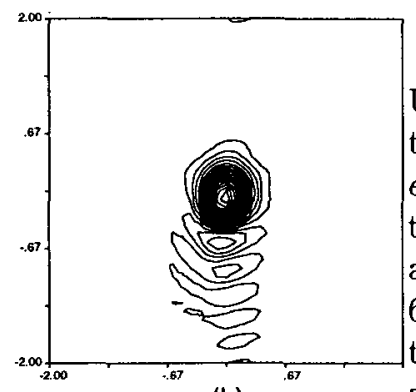

(b)
Figure 6: Four level scheme accuracy test result (a)advection along $\mathrm{x}$-direction (b)advection oblique to $\mathrm{x}$-direction, $\alpha=32.5^{\circ}$

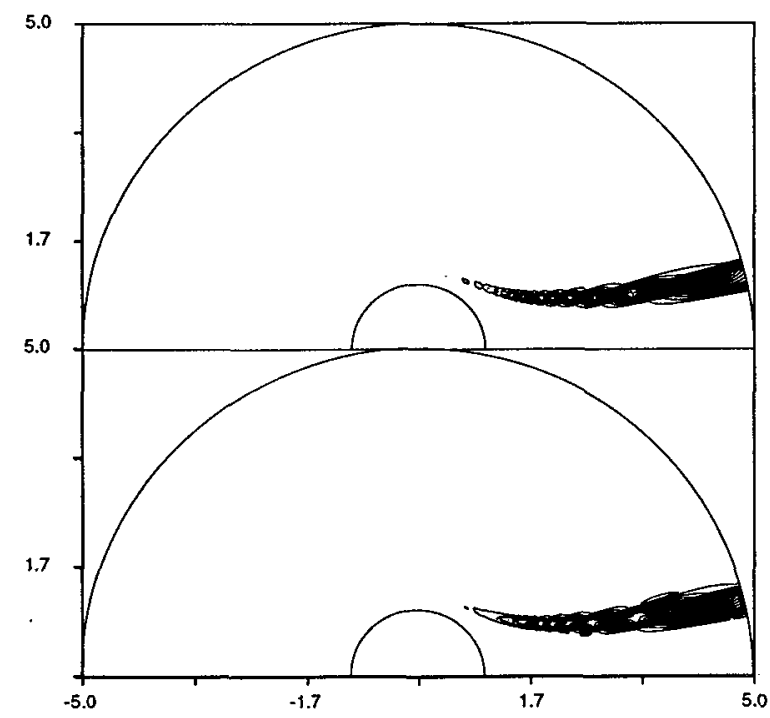

Figure 7: Scalar advection problem with creeping flow speed, $\mathrm{t}=14.0$, top:exact solution, bottom:numerical result where

$$
\begin{aligned}
& a_{r}=U_{\infty} \cos \left(1+\frac{a^{3}}{2 r^{3}}-\frac{3 a}{2 r}\right) \\
& a_{\theta}=U_{\infty} \sin \left(-1+\frac{a^{3}}{4 r^{3}}+\frac{3 a}{4 r}\right)
\end{aligned}
$$

Uniform velocity, $U_{\infty}$ and cylinder radius, $a$ are given the value 1.0. A Gaussian distribution $u(x, y)=$ $\exp \left(-\ln 2 / 0.3^{2} \cdot\left[(x+3)^{2}+(y-1)^{2}\right]\right)$ was given as an initial distribution. Computational domain was confined as $1<r<5$, and $0<\theta<\pi$. The grid size was $60 \times 90$. Numerical result is compared with the exact solution. Initial distribution was advected at different speed and stretched severely, Figure 7. Both solutions appear rather "lumpy" due to interpolation onto the coarse grid, but agreement between them is very clear.

\section{Two Dimensional Acoustics}

For two dimensional acoustics, the linearized Euler Equations without mean flow are used.

$$
\begin{aligned}
& p_{t}+u_{x}+v_{y}=0 \\
& u_{t}+p_{x}=0 \\
& v_{t}+p_{y}=0
\end{aligned}
$$

To mode the wave propagation precisely, characteristic equations are preferred.

$$
\begin{aligned}
& (p \pm u)_{t} \pm(p \pm u)_{x}+v_{y}=0 \\
& (p \pm v)_{t} \pm(p \pm v)_{y}+u_{x}=0
\end{aligned}
$$

where $q$ is a pressure stored with $y$-direction velocity, $v$. Equation 6 is aligned with $x$-direction and Equation 7 with $y$-direction. $(p \pm u)$ waves are mainly propagated along $x$-direction and $p$ and $u$ need to be updated and stored at same point. Again, $(p \pm v)$ waves are mainly transported along $y$-direction and $p$ and $v$ should be updated and stored at same point. Hence pressure is updated twice with different equations [3], it is stored at different points to avoid any dissipation or instability. To satisfy the criteria mentioned before, staggered grid, shown in Figure 8 is used in this study. The stencil shown on Figure 8.b is used to update the point where $(p, u)$ are stored and equations are discretized as below.

$$
\begin{aligned}
(p+u)_{a}= & (p+u)_{d 2}+(1-2 \nu)\left[(p+u)_{2}-(p+u)_{3}\right] \\
& -2 \mu\left(v_{5}-v_{7}\right) \\
(p-u)_{a}= & (p-u)_{d 1}+(1-2 \nu)\left[(p-u)_{2}-(p-u)_{1}\right] \\
& -2 \mu\left(v_{4}-v_{6}\right)
\end{aligned}
$$

where $\nu=\Delta t / \Delta x, \mu=\Delta t / \Delta y$. This is a three time level time reversible second order scheme for acoustics. It is compact and very simple to implement for given problem, but it requires a fine grid to resolve acoustic problem due to low order of accuracy. To improve phase 


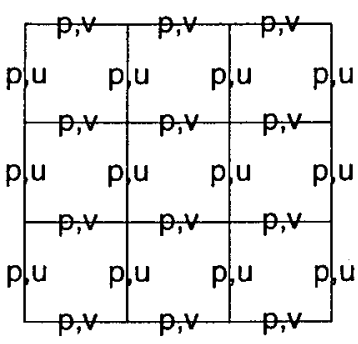

(a)

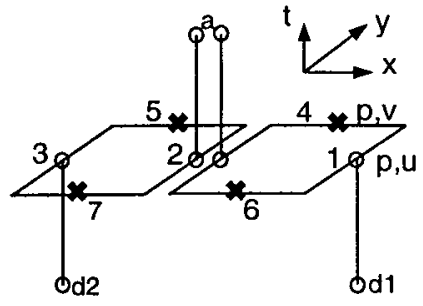

(b)
Figure 8: Illustrating staggered grid and three level stencil for second order upwind leapfrog scheme

properties, stencils are extended in space as shown in Figure 9. This is for $(p+u)$ wave computation. Obtaining a fourth-order scheme is straightforward. The second order scheme for $(p+u)$ wave is written with second order truncation term,

$$
\begin{aligned}
(p+u)_{a}=(p+u)_{c}+(1-2 \nu)\left[(p+u)_{6}-(p+u)_{7}\right] \\
-2 \mu\left(v_{6}-v_{7}\right)-\frac{\Delta t \Delta x^{2}}{6}(\nu-1)(2 \nu-1)\left(p_{x x x}+u_{x x x}\right) \\
-\frac{\Delta t^{2} \Delta x}{6}(2 \nu-3) q_{x y y}-\frac{\Delta t \Delta x^{2}}{12}\left(4 \nu^{2}-6 \nu+3\right) v_{x x y} \\
\quad-\frac{\Delta t^{3}}{3} u_{x y y}-\frac{\Delta t \Delta y^{2}}{12}(2 \mu-1)(2 \mu+1) v_{y y y}
\end{aligned}
$$

Then, discretizing the truncation errors and adding them to the left hand side increases the order of accuracy to four.

$$
\begin{aligned}
p_{x x x}= & {\left[\left(p_{5}-p_{8}\right)-3\left(p_{6}-p_{7}\right)\right] / \Delta x^{3} } \\
u_{x x x}= & {\left[\left(u_{5}-u_{8}\right)-3\left(u_{6}-u_{7}\right)\right] / \Delta x^{3} } \\
u_{x y y}= & \left(\frac{9}{8}\left[\left(u_{2}-2 u_{6}+u_{10}\right)-\left(u_{3}-2 u_{7}+u_{11}\right)\right]\right. \\
& \left.-\frac{1}{24}\left[\left(u_{1}-2 u_{5}+u_{9}\right)-\left(u_{4}-2 u_{8}+u_{12}\right)\right]\right) / \Delta x \Delta y^{2} \\
q_{x y y}= & {\left[\left(q_{1}-q_{2}-q_{3}+q_{4}\right)\right.} \\
& \left.-\left(q_{9}-q_{10}-q_{11}+q_{12}\right)\right] / 4 \Delta x \Delta y^{2} \\
v_{x x y}= & \left(\frac{9}{8}\left[\left(v_{2}-2 v_{6}+v_{10}\right)-\left(v_{3}-2 v_{7}+v_{11}\right)\right]\right. \\
& \left.-\frac{1}{24}\left[\left(v_{1}-2 v_{5}+v_{9}\right)-\left(v_{4}-2 v_{8}+v_{12}\right)\right]\right) / \Delta x^{2} \Delta y \\
v_{y y y}= & {\left[\left(v_{5}-v_{8}\right)-3\left(v_{6}-v_{7}\right)\right] / \Delta y^{3} }
\end{aligned}
$$

\subsection{Stable Discretization of Source Term}

In two dimensional acoustics, the linearized Euler equations in cylindrical coordinate include a source term, $-\frac{u}{r}$. Since simple handling of this term generates instabilities, special discretization for this term is necessary. This was studied with the simple spherical wave equation.

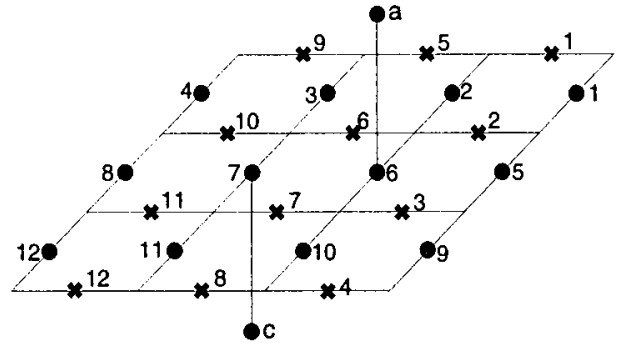

Figure 9: Three level stencil for fourth-order upwind leapfrog scheme

\subsubsection{One dimensional study}

$$
u_{t}+u_{r}=-\frac{u}{r}
$$

The spherical wave equation has a source term, $-\frac{u}{r}$. This equation could be simply discretized with the given stencil, Figure 10.

$$
u_{1}=u_{4}+\left(1-2 \frac{\Delta t}{\Delta r}\right)\left(u_{2}-u_{3}\right)-\frac{\Delta t}{r_{c}}\left(u_{2}+u_{3}\right)
$$

However, Fourier analysis reveals that the spurious mode is unstable. This unstable spurious mode excites an error that grows rapidly and overwhelms the physical mode. To avoid the instability, special handling of source term is necessary and we can rewrite the equation as follows.

$$
(r u)_{t}+(r u)_{r}=0
$$

Then it can be discretized stably as

$$
\begin{aligned}
& \frac{\left(r_{c}+\Delta r / 2\right)\left(u_{1}-u_{2}\right)+\left(r_{c}-\Delta r / 2\right)\left(u_{3}-u_{4}\right)}{2 \Delta t} \\
+ & \frac{\left(r_{c}+\Delta r / 2\right) u_{2}-\left(r_{c}-\Delta r / 2\right) u_{3}}{\Delta r}=0
\end{aligned}
$$

This second order scheme is stable and preserves the magnitude exactly. Simplifying the above equation leads to

$$
\begin{aligned}
u_{1}= & u_{4}+\left(1-2 \frac{\Delta t}{\Delta r}\right)\left(u_{2}-u_{3}\right)-\frac{\Delta t}{r_{c}}\left(u_{2}+u_{3}\right) \\
& -\frac{\Delta r}{2 r_{c}}\left(u_{1}-u_{2}-u_{3}+u_{4}\right)
\end{aligned}
$$

This stable scheme has an extra term compared with the unstable scheme, Equation 9 and this term turned out to be stabilizing the scheme.

\subsubsection{Two Dimensional Acoustics with Cylin- drical Coordinate}

To obtain a stable acoustics scheme, the governing equations are modified slightly in the way mentioned above.

$$
p_{t}+\frac{1}{r}(r u)_{r}+\frac{1}{r} v_{\theta}=0
$$



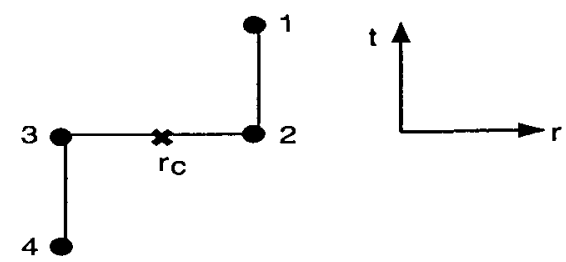

Figure 10: Three level stencil for one dimensional spherical wave equation

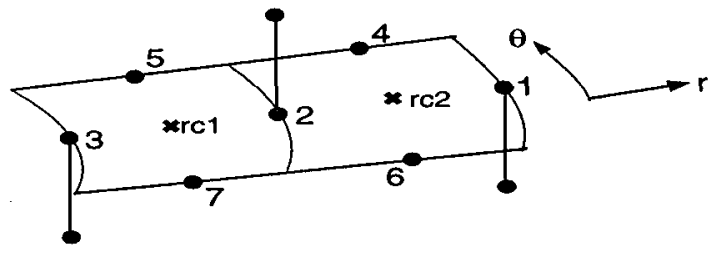

Figure 11: Second-Order stencil for two dimensional acoustics in cylindrical coordinate

$$
\begin{aligned}
& \frac{1}{r}(r u)_{t}+p_{r}=0 \\
& v_{t}+\frac{1}{r} p_{\theta}=0
\end{aligned}
$$

For discretization, these equations are written in characteristic form.

$$
\begin{aligned}
& {\left[p_{t} \pm \frac{1}{r}(r u)_{t}\right] \pm\left[p_{r} \pm \frac{1}{r}(r u)_{r}\right]+\frac{1}{r} v_{\theta}=0} \\
& {\left[p_{t} \pm v_{t}\right] \pm\left[p_{\theta} \pm v_{\theta}\right]+u_{r}+\frac{u}{r}=0}
\end{aligned}
$$

This manipulation does not have any effect on Equation 15 and the discretization of this equation does not need extra damping term. If Equation 14 is discretized on the stencil of Figure 11,

$$
\begin{gathered}
(p+u)_{2}^{n+1}=(p+u)_{3}^{n-1}+(1-2 \nu)\left[(p+u)_{2}^{n}-(p+u)_{3}^{n}\right] \\
-2 \mu\left(v_{5}^{n}-v_{7}^{n}\right)-\frac{\Delta r}{2 r_{c 1}}\left(u_{2}^{n+1}-u_{2}^{n}-u_{3}^{n}+u_{3}^{n-1}\right) \\
(p-u)_{2}^{n+1}=(p-u)_{1}^{n-1}+(1-2 \nu)\left[(p-u)_{2}^{n}-(p-u)_{1}^{n}\right] \\
-2 \mu\left(v_{4}^{n}-v_{6}^{n}\right)+\frac{\Delta r}{2 r_{c 2}}\left(u_{2}^{n+1}-u_{2}^{n}-u_{1}^{n}+u_{1}^{n-1}\right)
\end{gathered}
$$

This damping technique is applied to fourth-order schemes in the same way and a stable fourth-order acoustics scheme in cylindrical coordinate is obtained. To test this damping technique, an initial value problem was tested on a cylindrical grid including the origin. A pressure bump positioned at $(x=2.0, y=0.0)$ initially, passed the grid singularity at the origin without any kind of instability, Figure 12, as time evolved.

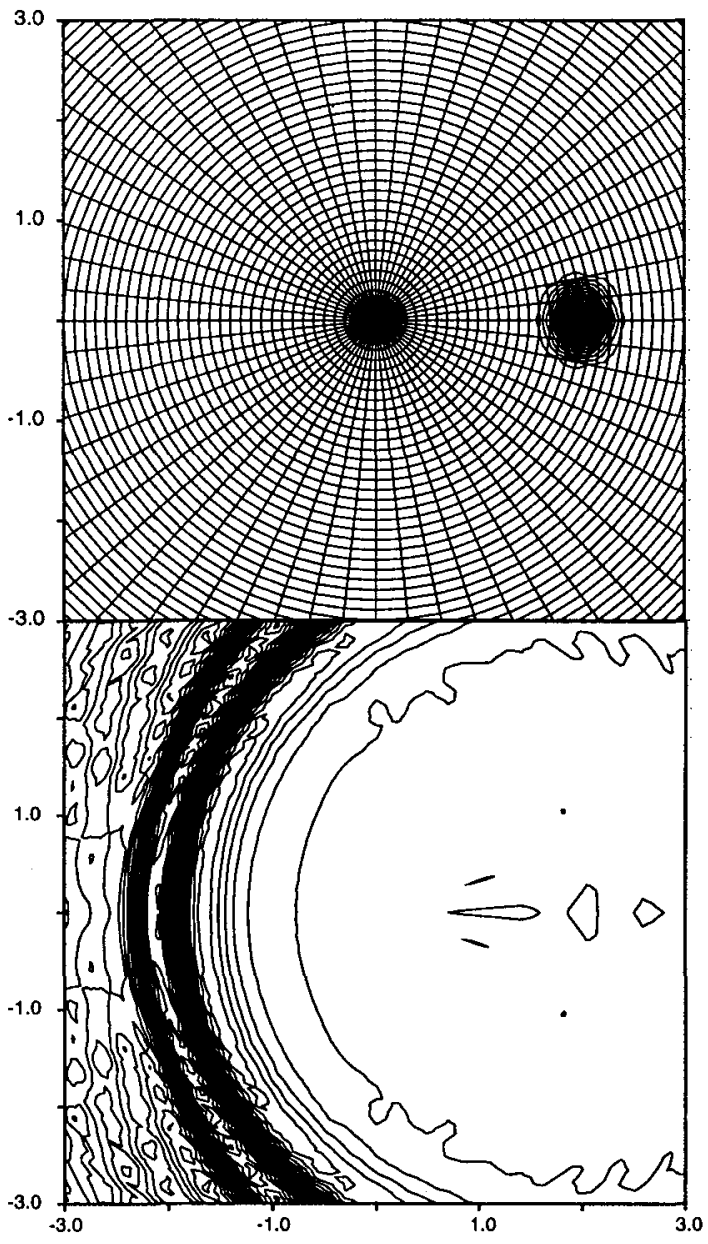

Figure 12: Test of damping technique with cylindrical coordinates including coordinate origin, top:initial distribution with grid, bottom:pressure contour at $\mathrm{t}=4.0$

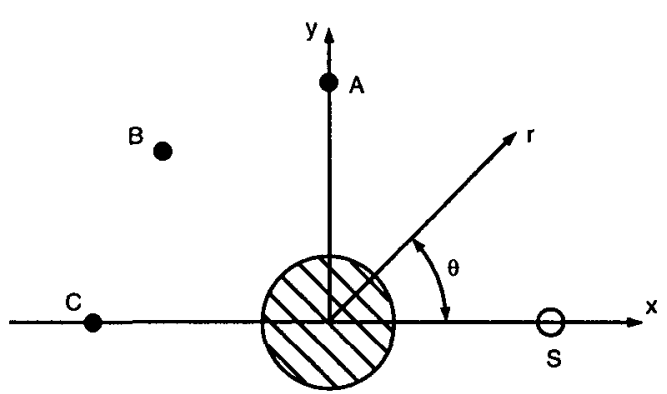

Figure 13: Explanation of acoustic scattering problem 
This problem is one of the second computational aeroacoustics benchmark problems[8]. An acoustic wave from the line source is scattered off the circular cylinder. This problem is a two dimension approximation to the sound field generated by a propeller scattered off the fuselage of an aircraft. Length is nondimensionalized by the diameter of circular cylinder, $D$, velocity by speed of sound, $c$, time by $D / c$, density by undisturbed density $\rho_{o}$ and pressure by $\rho_{o} c^{2}$. The linearized Euler equations are used as the governing equation.

$$
\begin{aligned}
& p_{t}+u_{r}+v_{\theta}+\frac{u}{r}=S \\
& u_{t}+p_{r}=0 \\
& v_{t}+\frac{1}{r} p_{\theta}=0
\end{aligned}
$$

where $u$ and $v$ are the disturbed velocities in radial and azimuthal direction. The 'line source' is represented by a rather narrow Gaussian source distribution.

$$
S=\exp \left[-\ln \left(\frac{(x-4)^{2}+y^{2}}{0.2^{2}}\right)\right] \sin \omega t, \quad \omega=8 \pi
$$

A huge grid was used for this computation. Ten grid points per wavelength were put in radial direction, $\Delta r / D=1 / 40$. The aspect ratio of the grid at the source was set to 1.0 and a small time step was used because of small grid size in azimuthal direction on the surface. Figure 14 shows the scattered pressure contour on the whole computational domain and figure 15 compares the numerical solution with the analytic one for $r \bar{p}^{2}$ at $r=15.0$. The numerical solution matches well with the analytic. The second problem is an initial value problem.

$$
S=\exp \left[-\ln \left(\frac{(x-4)^{2}+y^{2}}{0.2^{2}}\right)\right]
$$

Figure 16 shows pressure pulse contour at $t=6.0$. The reflected wave from the surface follows the pressure wave from the source directly. Pressure histories were checked at three points (A, B and $C)$ and showed in figure 17 . The solution of coarse $\operatorname{grid}(\Delta r / D=1 / 12)$ has little deviation from the analytic solution and other solutions are well matched with the analytic one.

\section{Two Dimensional Aeroacoustics}

Two dimensional linearized Euler equations with uniform mean flow are written in characteristic form.

$$
\begin{aligned}
& (p \pm u)_{t}+\left(M_{x} \pm 1\right)(p \pm u)_{x}+M_{y}(p+u)_{y}+v_{y}=0 \\
& (q \pm v)_{t}+M_{x}(q+v)_{x}+\left(M_{y} \pm 1\right)(q \pm v)_{y}+u_{x}=0
\end{aligned}
$$

where $M_{x}$ and $M_{y}$ are free stream Mach numbers in $x-$ and $y$-direction and $q$ is pressure stored with velocity $v$. For simplicity, developing the aeroacoustic scheme for $(p+u)$ wave is discussed mainly. Developing schemes for

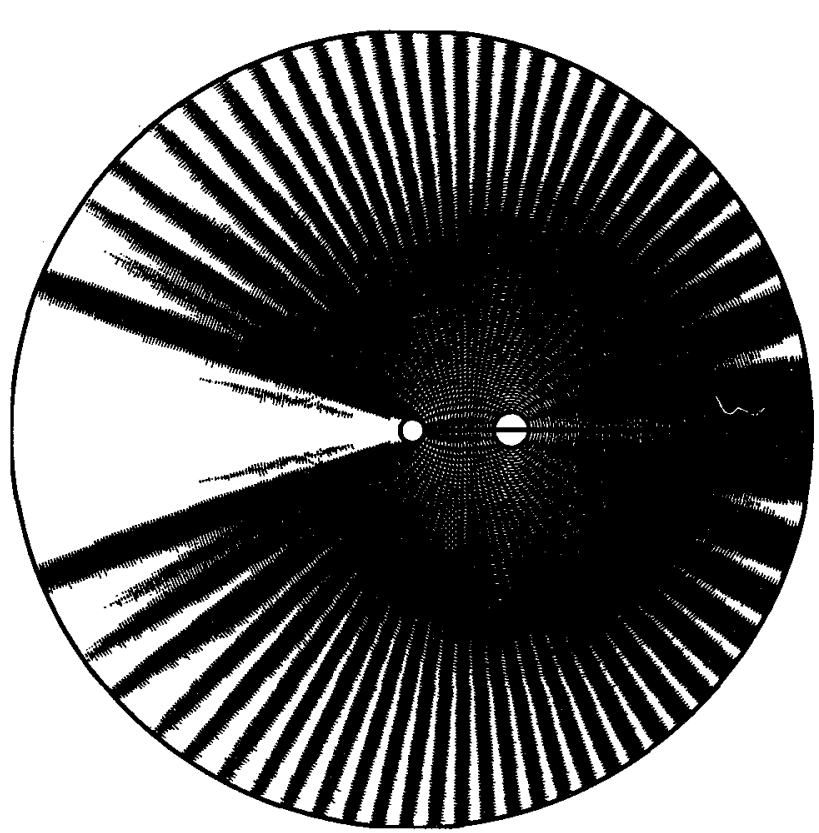

Figure 14: Acoustic scattering, $0.5<r<16.0$

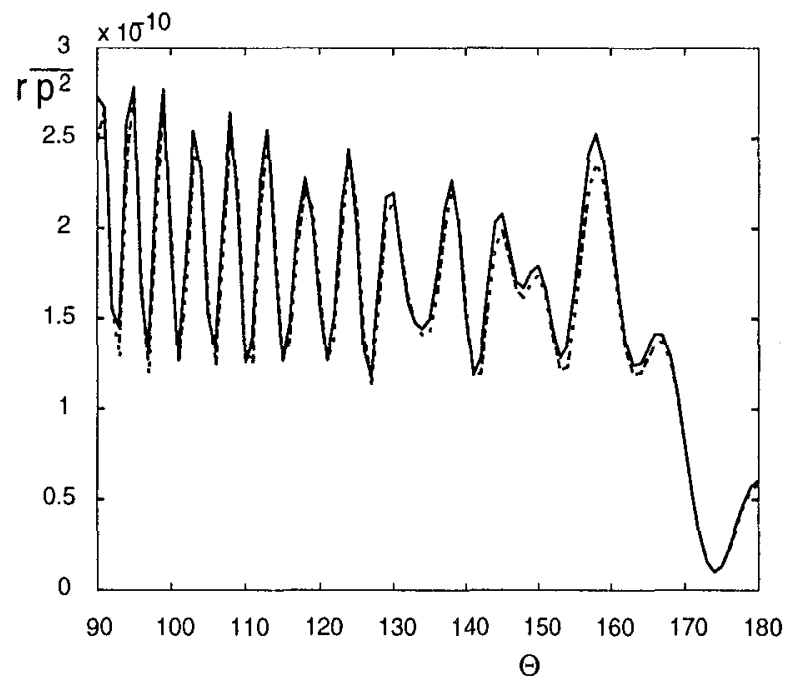

Figure 15: Comparison of directivity, solid line : numerical solution, dotted line : analytic solution, $r=15.0$ 


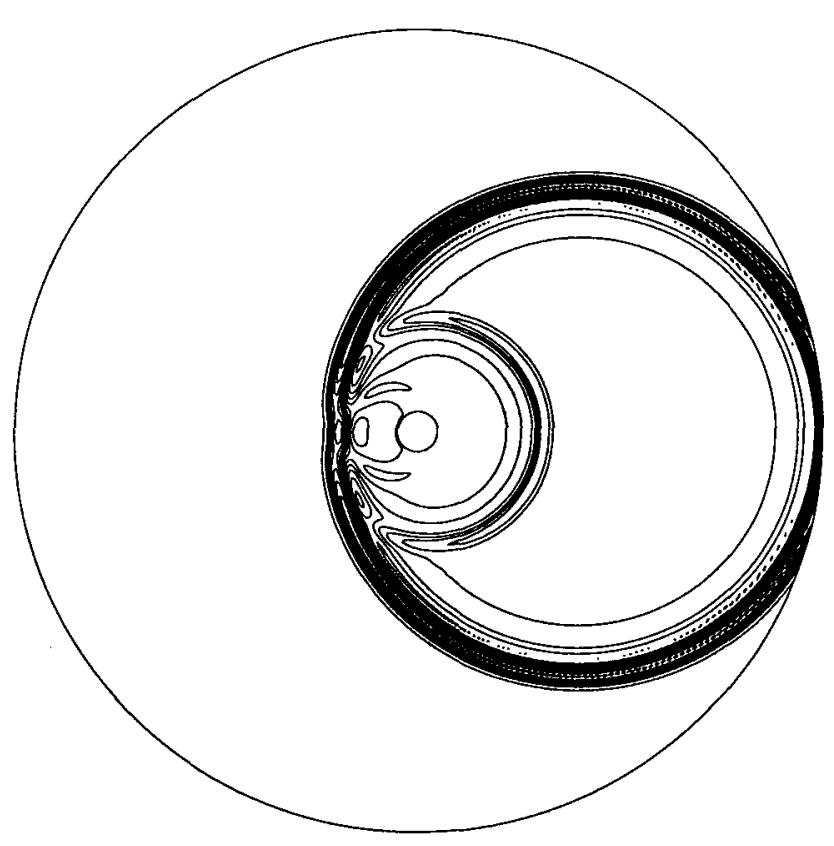

Figure 16: Pressure pulse contour of initial problem simulation, $0.5<r<10.0, \mathrm{t}=6.0$
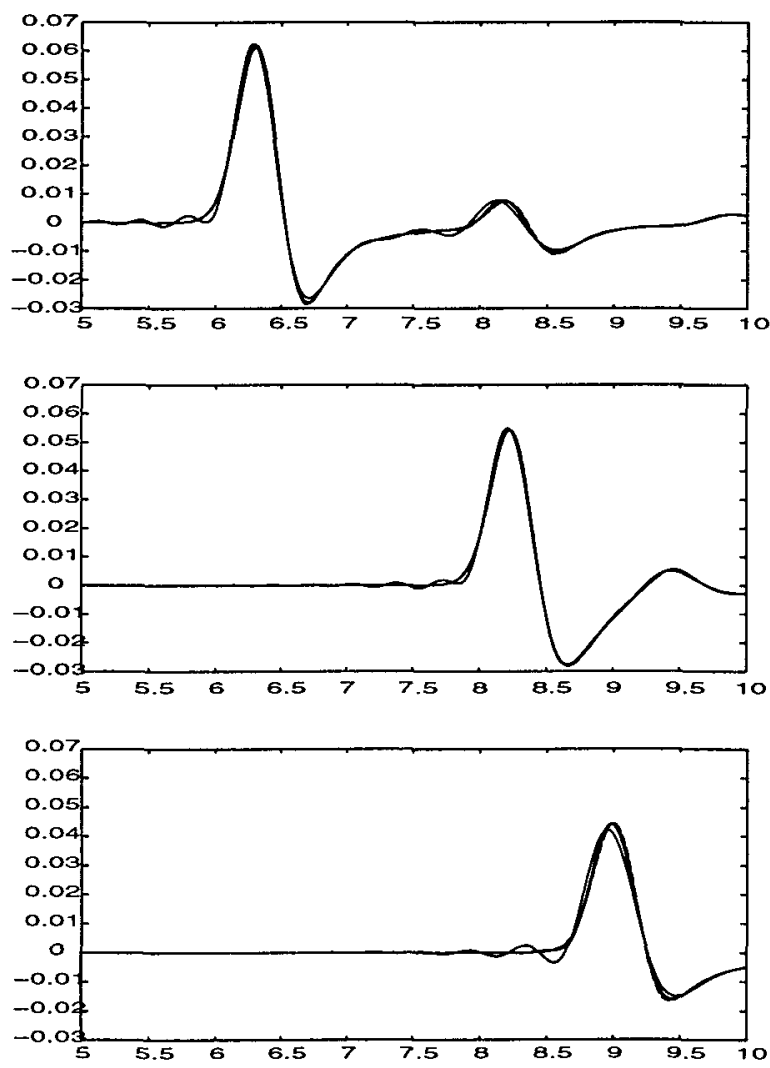

Figure 17: Pressure history at point A, B and C, dotted line : analytic solution, $\Delta r=1 / 12,1 / 16$ and $1 / 20$

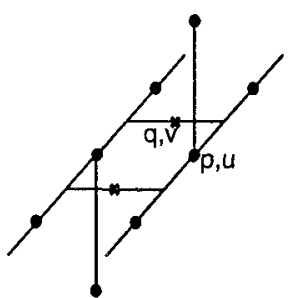

(a)

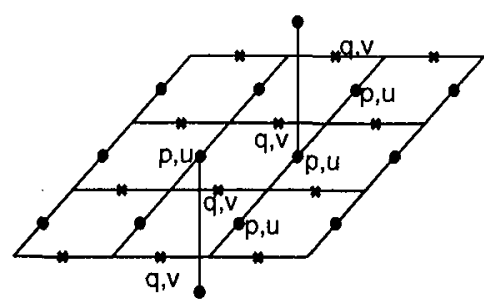

(b)
Figure 18: Second and fourth-order aeroacoustics schemes (a)second-order scheme, (b)fourth-order scheme

where $M_{x}$ and $M_{y}$ are free stream Mach numbers in $x-$ and $y$-direction and $q$ is pressure stored with velocity $v$. For simplicity, developing the aeroacoustic scheme for $(p+u)$ wave is discussed mainly. Developing schemes for other variable, $(p-u)$ and $(q \pm v)$ are very similar to that of $(p+u)$ and omitted here. Since characteristic variable, $(p+u)$ is advected in $x$-direction and also $y$-direction, second-order compact scheme is not possible and stencil must be extended in $y$-direction, Figure 18.a.

\subsection{Fourth-order upwind leapfrog scheme for aeroacoustics}

To increase the order of accuracy to four, the stencil for the second-order scheme is extended in space, Figure 18.b. Discretizing the characteristic equation on this stencil does not achieve the fourth-order accuracy and its modified equation has a couple of second-order truncation terms.

$$
\begin{aligned}
& \sum C_{i} U_{i}=(p+u)_{t}+\left(M_{x}+1\right)(p+u)_{x}+M_{y}(p+u)_{y} \\
& +v_{y}+\Delta x^{2} M_{y}\left(1+\nu^{2} M_{y}^{2}\right) / 6\left(p_{y y y}+u_{y y y}\right)+O\left(\Delta x^{4}\right)
\end{aligned}
$$

We still need to discretize $p_{y y y}$ and $u_{y y y}$ to obtain fourthorder accuracy. Discretizing these terms directly requires a larger stencil which makes it hard to implement the scheme, especially at domain boundaries. Therefore discretizing the second-order truncation terms without extending the stencil is explained in the following section.

\subsubsection{Discretization of $p_{y y y}$}

At least four different points of $p$ are necessary to discretize $p_{y y y}$. On the stencil, Figure 18.b, however, only three different points of $p$ are available in $y$-direction and it is not possible to discretize $p_{y y y} . q$ is also a pressure stored with velocity in $y$-direction, $v$ and four points are available in $y$-direction. With these four $q$ points, we can discretize $q_{y y y}$. Therefore, replacing $p_{y y y}$ with $q_{y y y}$ and discretizing can eliminate one of the secondorder truncation terms. However, simply replacing $p_{y y y}$ with $q_{y y y}$ turned out to be unstable. It was better to 
for aeroacoustics in this paper are.

$(p \pm u)_{t}+\left(M_{x} \pm 1\right)(p \pm u)_{x}+M_{y}(q \pm u)_{y}+v_{y}=0(19)$

$(q \pm v)_{t}+M_{x}(p \pm v)_{x}+\left(M_{y} \pm 1\right)(q \pm v)_{y}+u_{x}=0(20)$

$p_{y}$ is replaced by $q_{y}$ in Equation 19 and $q_{x}$ by $p_{x}$ in Equation 20.

\subsubsection{Discretization of $u_{y y y}$}

There is no precise equivalent to the above trick. However, if we assume that the vorticity field is known at each edge, we can replace $u_{y y y}$ with $\omega_{y y}$ and $v_{x y y}$.

$$
u_{y y y}=v_{x y y}-\omega_{y y}
$$

$v_{x y y}$ can be discretized with the given stencil and $\omega_{y y}$ is found simply. For the example given next, $\omega \equiv 0$ initially and will remain so. For the aeroacoustic problem, with a uniform background flow, any vorticity present in the perturbation is a convected quantity. We would be able to compute it using the methods of section 2 .

\subsection{Sixth-order upwind leapfrog scheme}

Increasing the order of accuracy to six was attempted by extending the stencil in space in the way described before. The discretized equation again has a fourthorder truncation term, $u_{\text {yyyyy }}$ and fully sixth-order aeroacoustic schemes are obtained by replacing the truncation term with $v_{x y y y}$ and $\omega_{y y y y}$.

$$
u_{y y y y y}=v_{x y y y y}-\omega_{y y y y}
$$

\subsection{Numerical experiment - Reflection of an acoustic pulse}

For the numerical experiment, reflection of an acoustic pulse off a wall in the presence of a uniform flow in semiinfinite space was considered, figure 19. The linearized Euler equations in characteristic form are

$$
\begin{aligned}
& (p \pm u)_{t}+(M \pm 1)(p \pm u)_{x}+v_{y}=0 \\
& (q \pm v)_{t}+M(p \pm v)_{x} \pm(q \pm v)_{y}+u_{x}=0
\end{aligned}
$$

where $M$ is free stream Mach number and $q$ is again another pressure stored with $v$. In this experiment, four different schemes are tested and the results are compared with the analytic one. The first one was 'nearfourth-order';it uses the setncil of figure 18 .b but does not include the additional corrections discussed in 4.1.2, the second was a fourth-order one with equation 21 , the third was a fourth-order scheme with larger stencil and the last was a sixth-order one. In this example, there was no initial vorticity distribution and vorticity remained zero during time evolution.

$$
\begin{aligned}
& t=0, u=v=0 \\
& p=\exp \left(-\ln 2\left[\frac{x^{2}+(y-25)^{2}}{25}\right]\right)
\end{aligned}
$$

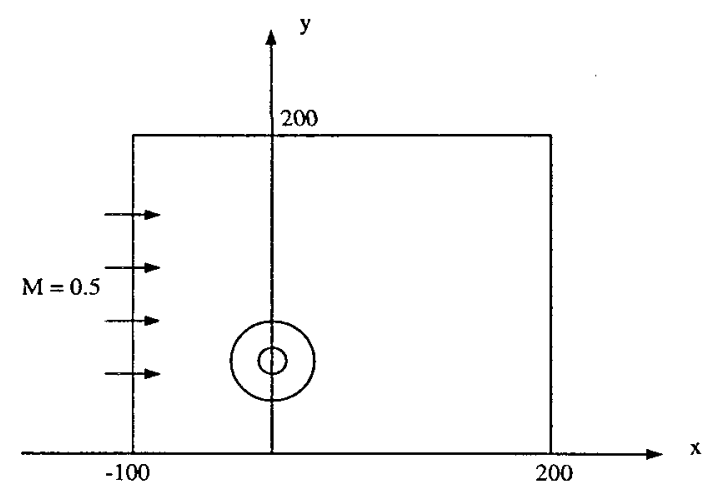

Figure 19: Illustration of example for aeroacoustics schemes

The pressure wave propagates in all direction while being also advected in $x$-direction. Figure 20 shows pressure contours at three different times. Figure 21 shows the numerical solutions with the analytic one. The analytic solution is represented merely by its grid-point values, so that the peaks are clipped by linear interpolation in the plots. Pressure profiles at $x=50$ along $y$-direction at $t=100$ are compared. On the finest grid, $\Delta x=2.0$, all schemes performed very well. On a slightly coarser grid, $\Delta x=2.5$, the second-order scheme begins to show significant errors in certain regions. On the coarest grid, $\Delta x=5.0$, both the sixth-order and wide-stencil fourthorder schemes continue to do well. None of the schemes, however, gave meaningful results with $\Delta x=10.0$ and we do not show these results.

\section{Conclusion}

The upwind leapfrog method which is time reversible and upwind biased, was multidimensionalized successfully for advection, acoustic and aeroacoustics with edge-based storage. A range of test problems have demonstrated accurate solutions on coarse grids. Since, however, higher order schemes with space-extended stencils require special treatment at boundaries(wall, far-field), more studies for higher order method adaptable to both boundaries are necessary.

\section{References}

[1] J.P. Thomas and P.L. Roe, Development of nondissipation numerical schemes for computational aeroacoustics, In AIAA 11th Computational Fluid Dynamics Conference, 1993

[2] J.P. Thomas, C. Kim and P.L. Roe, Progress towards a new Computational scheme for aeroacoustics, In AIAA 12th Computational Fluid Dynamics Conference, 1995 
[3] P.L. Roe, Linear bicharacteristic schemes without dissipation, SIAM Journal Scientific Computation to appear also ICASE Report 94-65, 1994

[4] A. Iserles, Generalised leapfrog methods, IMA Journal of Numerical Analysis, 6, 1986

[5] J.P. Thomas, An investigation of the upwind leapfrog method for scalar advection and acoustic/aeroacoustic wave propagation problems, Ph.D. Thesis, Department of Aerospace Engineering, University of Michigan, 1996

[6] B.T. Nguyen, Investigation of three-level finitedifference time-domain methods for multidimensional acoustics and electromagnetics, Ph.D. Thesis, Department of Aerospace Engineering, University of Michigan, 1996

[7] Proceeding of ICASE/LaRC 1st workshop on benchmark problems in Computational Aeroacoustics, edited by J.C. Hardin, J.R. Ristorcelli and C.K.W. Tam, NASA CP-3300

[8] Proceeding of ICASE/LaRC 2nd workshop on benchmark problems in Computational Aeroacoustics, in preparation

[9] S.K. Lele, Compact finite difference schemes with spectral-like resolution, Journal of Computational Physics, 103:16-42, 1991

[10] C.K.W. Tam and J.C. Webb, Dispersion-relationpreserving finite difference schemes for computational acoustics, Journal of Computational Physics, 107:262-281, 1992

[11] R.Hixon,Evaluation of a high-accuracy MacCormack-type scheme using benchmark problems, NASA CR-202324

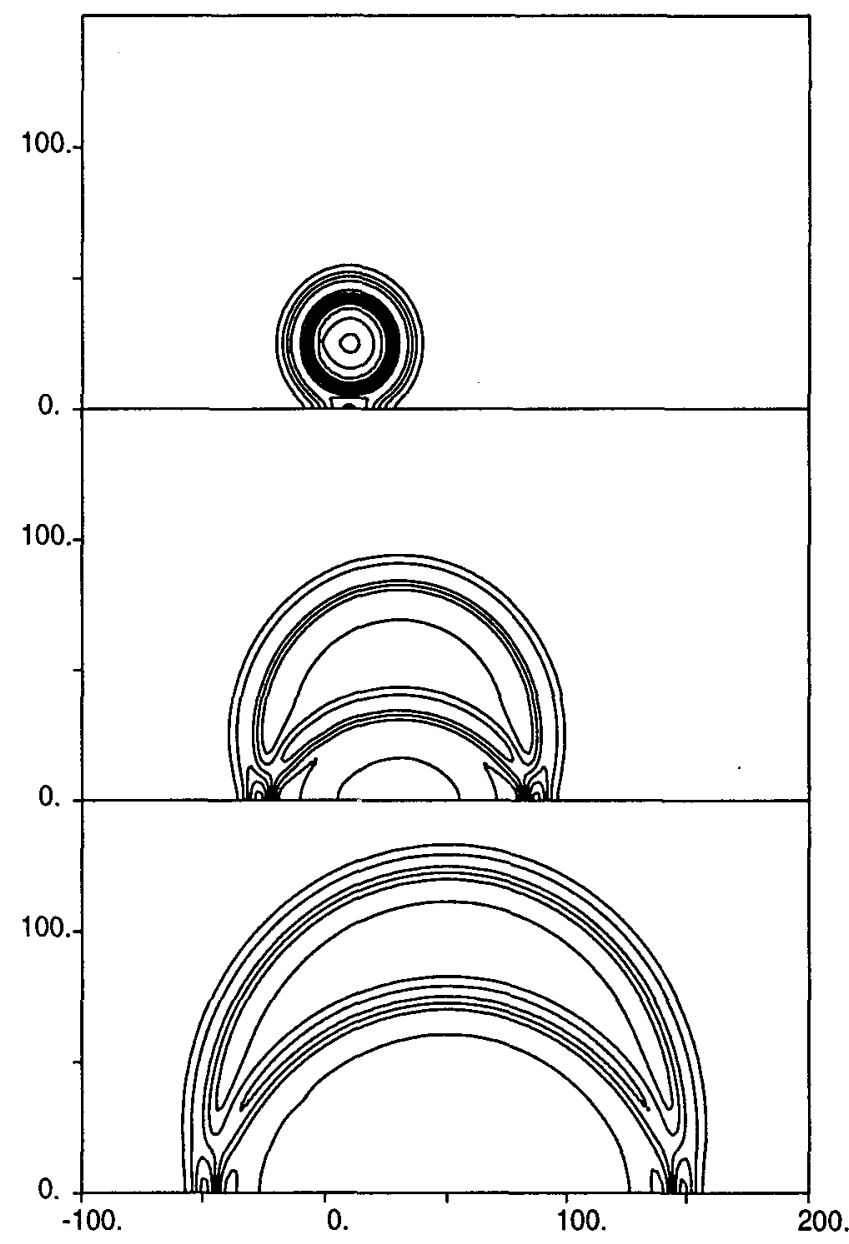

Figure 20: Pressure pulse contours at different times, top $: \mathrm{t}=20.0$, middle $: \mathrm{t}=60.0$, bottom $: \mathrm{t}=100.0$ 


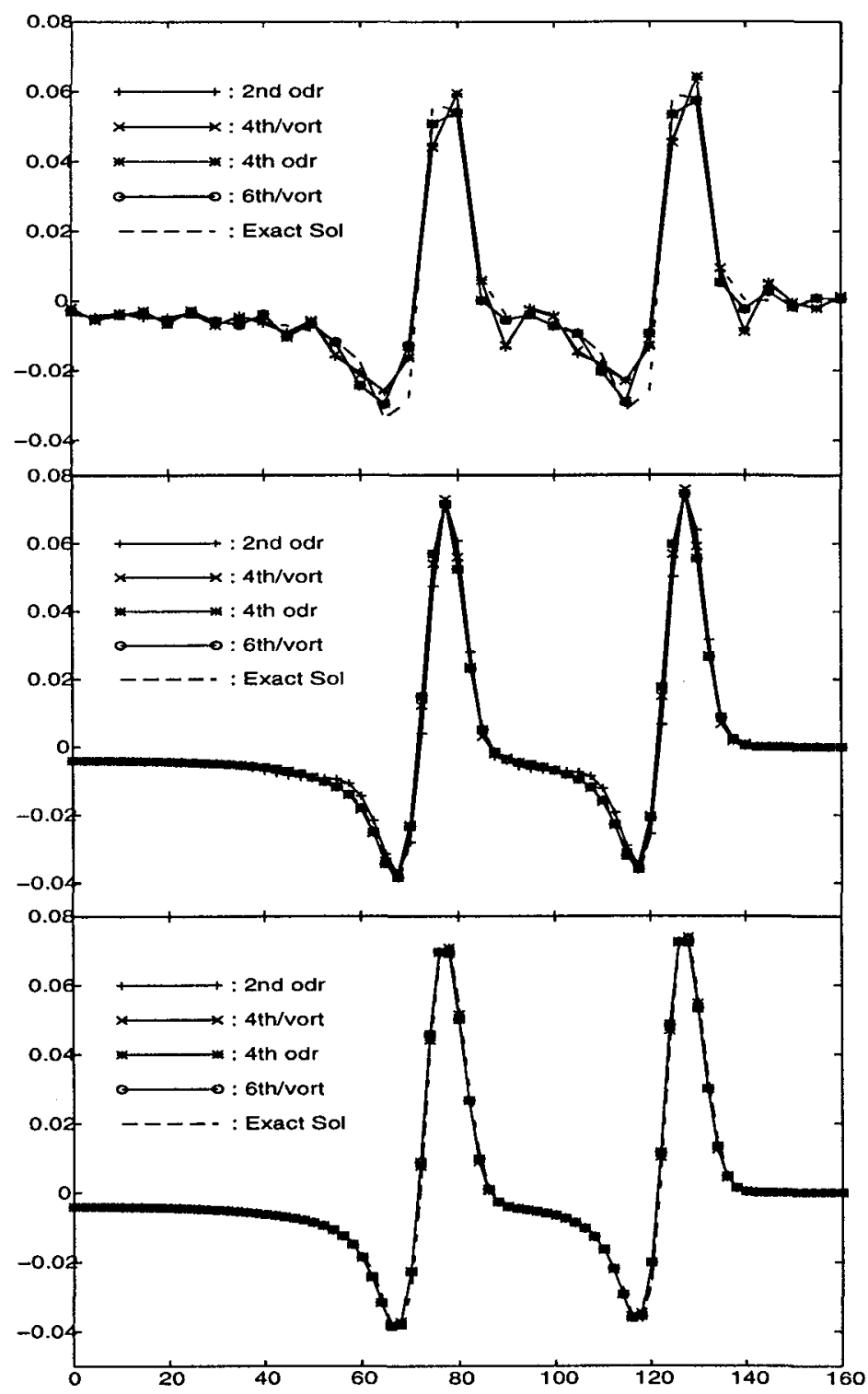

Figure 21: Pressure profiles along $y$-axis at $x=50$, $\mathrm{t}=100$, top : $\Delta \mathrm{x}=5.0$, middle : $\Delta \mathrm{x}=2.5$, bottom : $\Delta \mathrm{x}=2.0$ 